\title{
全叶马兰中两个新的黄酮类化合物
}

\author{
王国凯 ${ }^{a}$ 孙云鹏 ${ }^{a}$ 王 诤 ${ }^{a}$ 张 楠 ${ }^{a}$ 王 刚*,a \\ 周忠玉 $b$ 刘劲松*,a \\ ( ${ }^{a}$ 安徽中医药大学药学院 现代中药安徽省重点实验室 合肥 230012) \\ $\left({ }^{b}\right.$ 中国科学院华南植物园 广东省应用植物学重点实验室 广州 510650)
}

\begin{abstract}
摘要 利用多种色谱分离方法对全叶马兰(Kalimeris integrifolia) 的化学成分进行研究, 从其地上部分的乙醇提取物中 分离得到两个化合物, 应用 HR-ESIMS, 1D 和 2D NMR, IR, UV 等多种波谱技术确定了化合物的化学结构, 经鉴定分别 为 5,8-二羟基-2-(3-羟基-4,5-二甲氧苯基)-6,7-二甲氧基-4 $\mathrm{H}$-色烯-4-酮(1)和 5-羟基-6,7-二甲氧基-2-(4'-甲氧基苯基)-4-氧 代- $4 H$-色烯-8-(2"-甲基丁酸酯) (2). 化合物 $\mathbf{1}$ 和 2 为两个新的黄酮类化合物.
\end{abstract}

关键词 全叶马兰; 菊科; 化学成分; 黄酮类

\section{Two New Flavonoids from Kalimeris integrifolia}

\author{
Wang, Guokai ${ }^{a} \quad$ Sun, Yunpeng ${ }^{a} \quad$ Wang, Zheng $^{a} \quad$ Zhang, Nan $^{a} \quad$ Wang, Gang ${ }^{* a}$ \\ Zhou, Zhongyu $^{b} \quad$ Liu, Jinsong ${ }^{*, a}$ \\ ( ${ }^{a}$ Anhui Key Laboratory for mordern Chinese Materia Medica, School of Pharmacy, Anhui University of \\ Chinese Medicine, Hefei 230012) \\ ( ${ }^{b}$ Guangdong Provincial Key Laboratory of Applied Botany, South China Botanical Garden, \\ Chinese Academy of Sciences, Guangzhou 510650)
}

\begin{abstract}
Investigation on Kalimeris integrifolia resulted in two compounds by means of various chromatographic techniques (silica gel, Sephadex LH-20, RP-LC and Pre-HPLC). Their structures were determined as 5,8-dihydroxy-2-(3-hydroxy4,5-dimethoxyphenyl)-6,7-dimethoxy-4H-chromen-4-one (1) and 5-hydroxy-6,7-dimethoxy-2-(4-methoxyphenyl)-4-oxo-4Hchromen-8-yl 2-methylbutanoate (2) on the basis of extensive spectroscopic analyses including MS, IR, UV, 1D- and 2DNMR. Among them, compounds $\mathbf{1}$ and $\mathbf{2}$ are two new flavonoids, named as kalinturflavone A (1) and kalinturflavone B (2).
\end{abstract}

Keywords Kalimeris integrifolia; Asteraceae; chemical constituent; flavonoids

全叶马兰为菊科马兰属植物 (Kalimeris integrifolia Turcz.ex DC.), 别名全缘叶马兰、野白菊(湖北)、黄花三 草、扫帚花、扫帚鸡肠、全叶紫苑, 主要生于山坡、林 缘、灌从、路旁, 我国大部分地区均有分布, 也分布于 朝鲜、日本、西伯利亚东部 ${ }^{[1]}$. 《中华本草》 ${ }^{[2]}$ 中记载, 全 叶马兰味苦、性寒, 具有清热解毒、止咳、散瘀止血的 功效. 至今为止, 没有文章报道过全叶马兰的次级代谢 产物的研究, 本课题组近年一直从事马兰相关研究, 从 马兰中分离并成分包括甾体、萜类、黄酮、酚酸等多种 类型, 并进行了肝细胞保护及抗肿瘤活性研究 ${ }^{[3 \sim 6]}$. 为
了更加深入地研究马兰属植物的次生代谢产物, 阐明全 叶马兰和马兰属各物种间的化学成分差异性, 我们对全 叶马兰进行了化学成分研究, 从中分离纯化得到 2 个新 的黄酮化合物，运用 HR-ESIMS, 1D 和 2D NMR 等光谱 技术确定新化合物的结构为 Kalinturflavone A (1) 和 Kalinturflavone B (2)(图 1). 黄酮类化合物具有抑制 MMP-9 的生物活性 ${ }^{[7]}$. 本研究分别利用五种肿瘤细胞株 HL-60, SMMC-7721, A-549, MCF-7 和 SW480 对所分离 得到的两个黄酮 1 和 2 进行活性测试, 但两者活性都很 弱 $\left(\mathrm{IC}_{50}>40 \mu \mathrm{mol} / \mathrm{L}\right)$.

* Corresponding authors. E-mail: jinsongliu108@sina.com; kunhong_8@163.com

Received November 18, 2016; revised December 13, 2016; published online January 17, 2017.

Project supported by the National Natural Science Foundation of China (No. 81303220), the Natural Sciences Foundation of Anhui Province (No. 11040606M220) and the Guangdong Provincial Key Laboratory of Applied Botany, South China Botanical Garden, CAS (No. AB2015001).

国家自然科学基金(No. 81303220)、安徽省自然科学基金(No. 11040606M220)和中国科学院华南植物园广东应用植物重点实验室(No. AB2015001)资 助项目. 


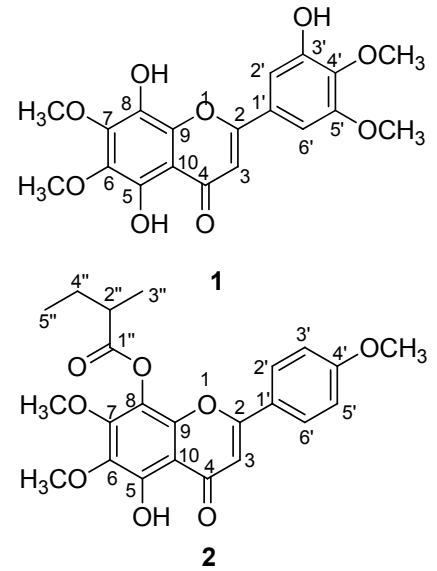

图 1 化合物 1 和 2 的结构图

Figure 1 Structures of compounds 1 and 2

\section{1 结果与讨论}

\section{1 化合物 1 的结构鉴定}

黄色针状结晶(甲醇), HR-ESI-MS 给出化合物的分 子式为 $\mathrm{C}_{19} \mathrm{H}_{18} \mathrm{O}_{9}\left(\mathrm{~m} / \mathrm{z} 389.0894[\mathrm{M}-\mathrm{H}]^{-}\right.$, 计算值为 $389.0878)$, 提示有 11 个不饱和度. 在 IR 光谱中, 3419、 1657、1615 和 $1594 \mathrm{~cm}^{-1}$ 处吸收带显示, 该化合物依次 存在羟基、羰基、苯环, 经 UV 光谱 $\left(\lambda_{\max }\right)$ 在 $306 \mathrm{~nm}$ 吸 收带, 结合盐酸一镁粉反应阳性初步推断该化合物为一 黄酮类化合物. 由 ${ }^{1} \mathrm{H} N \mathrm{NMR}$ 谱中低场单峰 $\delta 12.35(\mathrm{~s}, 1 \mathrm{H})$ 显示此峰为羟基与羰基形成氢键后所出的峰, 通过 HSQC 二维相关谱发现氢谱中 $\delta 6.98(\mathrm{~s}, 1 \mathrm{H})$ 与碳谱中 $\delta 104.2$ (C-3)峰相关, 所以确定此峰为 C环 3 号位的氢信 号, 可进一步确定该化合物为黄酮类化合物; 氢谱中低 场两个单峰信号 $\delta 9.69(\mathrm{~s}, 1 \mathrm{H})$ 及 $9.33(\mathrm{~s}, 1 \mathrm{H})$ 为两个羟基 信号, 两个双峰 $\delta 7.31(\mathrm{~d}, J=1.6 \mathrm{~Hz}, 1 \mathrm{H})$ 和 $7.24(\mathrm{~d}, J=$ $1.6 \mathrm{~Hz}, 1 \mathrm{H})$ 显示为黄酮 $\mathrm{AB}$ 系统中 $\mathrm{B}$ 环上 $2^{\prime}-\mathrm{H}$ 和 $6^{\prime}-\mathrm{H}$ 信号, 并且由于 $\mathrm{H}-2^{\prime}$ 和 H-6'信号未重叠, 所以可知 B 环 上的取代基不对称，因此可知 $3^{\prime}$ 和 $5^{\prime}$ 位的取代基分别为 差基和甲氧基, 这一点也可以通过 $\mathrm{HSQC}$ 和 $\mathrm{HMBC}$ 二 维相关谱进行验证. 由氢谱中高场区四个单峰信号 $\delta 3.95(\mathrm{~s}, 3 \mathrm{H}), 3.89(\mathrm{~s}, 3 \mathrm{H}), 3.83(\mathrm{~s}, 3 \mathrm{H}), 3.76(\mathrm{~s}, 3 \mathrm{H})$ 可知 此化合物有四个甲氧基, 并结合上述结论可知 $\mathrm{A}$ 环全部 被取代, 通过 HMBC 二维相关谱可知 $\delta 3.83(\mathrm{~s}, 3 \mathrm{H}$, $\left.\mathrm{OCH}_{3}\right)$ 与 $136.2(\mathrm{C}-6)$ 相关, $3.95\left(\mathrm{~s}, 3 \mathrm{H}, \mathrm{OCH}_{3}\right)$ 与 148.1 $(\mathrm{C}-7)$ 相关, $3.76\left(\mathrm{~s}, 3 \mathrm{H}, \mathrm{OCH}_{3}\right)$ 与 $139.8\left(\mathrm{C}-4^{\prime}\right)$ 相关, 3.89 $\left(\mathrm{s}, 3 \mathrm{H}, \mathrm{OCH}_{3}\right)$ 与 $153.5\left(\mathrm{C}-5^{\prime}\right)$ 相关, 并且 $12.35(\mathrm{~s}, 1 \mathrm{H}$, $\mathrm{OH})$ 与 $144.6(\mathrm{C}-5), 136.2(\mathrm{C}-6), 106.5(\mathrm{C}-10)$ 相关, 9.33 $(\mathrm{s}, 1 \mathrm{H}, \mathrm{OH})$ 与 $148.1(\mathrm{C}-7), 130.7(\mathrm{C}-8), 141.4(\mathrm{C}-9)$ 相关, 所以 6、7、 $4^{\prime}$ 及 $5^{\prime}$ 位上均为甲氧基; $9.33(\mathrm{~s}, 1 \mathrm{H}, \mathrm{OH})$ 与 $130.7(\mathrm{C}-8)$ 相关, $9.69(\mathrm{~s}, 1 \mathrm{H}, \mathrm{OH})$ 与 $150.9\left(\mathrm{C}-3^{\prime}\right)$ 相关, 所 以 8 和 3 '位为羟基(图 2). 综上所述, 并与文献[ $8 \sim 12]$
对照，确定该化合物为 5,8 二羟基-2-(3-羟基- 4,5-二甲氧 苯基)-6,7-二甲氧基-4H-色烯-4-酮 (图 1)，命名为 Kalintur-flavone A, 其 ${ }^{1} \mathrm{H}$ NMR、 ${ }^{13} \mathrm{C}$ NMR 数据见表 1 .

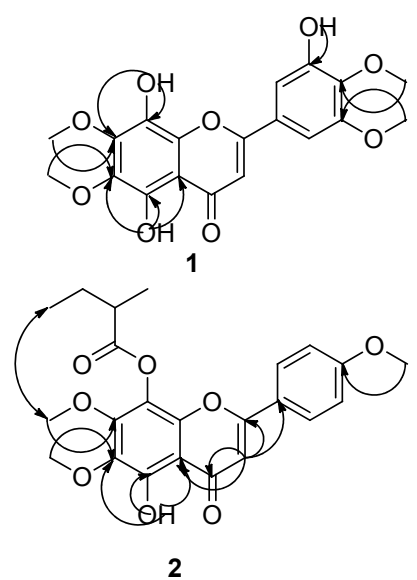

图 2 化合物 $\mathbf{1}$ 和 $\mathbf{2}$ 的主要 $\mathrm{HMBC}(\leftrightharpoons)$ 和 ROESY ( $)$ 相关 Figure 2 Main HMBC ( $\frown$ ) and ROESY ( $\rightleftharpoons$ ) relevant of compounds $\mathbf{1}$ and $\mathbf{2}$

\section{2 化合物 2 的结构鉴定}

黄色粉末(甲醇), HR-ESI-MS 给出化合物的分子式 为 $\mathrm{C}_{23} \mathrm{H}_{24} \mathrm{O}_{8}(\mathrm{~m} / z 427.1399 \text { [M-H] }]^{-}$, 计算值为 427.1398), 提示有 12 个不饱和度. 通过红外光谱、质谱、氢谱可初 步了解该化合物与化合物 1 母核结构类似，都是黄酮类 化合物，该化合物的氢谱碳谱与化合物 $\mathbf{1}$ 不同的是少了 一个甲氧基、两个羟基，多了两个甲基、一个亚甲基，一 个次甲基和一个羰基. 由 ${ }^{1} \mathrm{H}$ NMR 谱中 $\delta 7.76(\mathrm{~d}, J=8.8$ $\mathrm{Hz}, 2 \mathrm{H}), 6.98(\mathrm{~d}, J=8.8 \mathrm{~Hz}, 2 \mathrm{H})$ 表示 B 环为单取代苯环, 并由 $\mathrm{HMBC}$ 二维相关谱可知 $\delta 3.96\left(\mathrm{~s}, 3 \mathrm{H}, \mathrm{OCH}_{3}\right)$ 与 $136.5(\mathrm{C}-6)$ 相关, $4.02\left(\mathrm{~s}, 3 \mathrm{H}, \mathrm{OCH}_{3}\right)$ 与 $151.7(\mathrm{C}-7)$ 相关, $3.88\left(\mathrm{~s}, 3 \mathrm{H}, \mathrm{OCH}_{3}\right)$ 与 $162.7\left(\mathrm{C}-4^{\prime}\right)$ 相关，由此相关可知 3 个甲氧基的位置; 再由 $\mathrm{HSQC}$ 和 ${ }^{1} \mathrm{H}-{ }^{1} \mathrm{H}$ COSY 二维相关 谱可将两个甲基、一个亚甲基，一个次甲基和一个羰基 链接起来, 形成一个 2-甲基丁酸酯基团, 基于 $\mathrm{A}$ 环上的 氢除 8 位外全部被取代, 所以此链只能在 8 位与黄酮母 核成酯，并且通过 ROESY 二维相关谱可看到 $5^{\prime \prime}$ 位甲基 氢与 7 位甲氧基上的氢有相关(图 2). 综上所述，并与文 献[13，14]对照，确定该化合物为 5-羟基-6,7-二甲氧基2-(4'-甲氧基苯基)-4-氧代-4H-色烯-8-(2"-甲基丁酸酯) (图 1), 命名为 Kalinturflavone B, 其 ${ }^{1} \mathrm{H}$ NMR、 ${ }^{13} \mathrm{C}$ NMR 数据见表 1 .

\section{2 实验部分}

\section{1 仪器与试剂}

质谱由 Waters AutoSpec Premier P776 和 Xevo TQ-S 质谱仪测定 (美国Waters 公司生产); 紫外光谱由 
表 1 化合物 $1\left(600 / 150 \mathrm{MHz}, \mathrm{DMSO}-d_{6}\right)$ 和 $2(600 / 150 \mathrm{MHz}$, $\left.\mathrm{CDCl}_{3}\right)$ 的 $\mathrm{NMR}$ 数据

Table 1 NMR data of compounds $1\left(600 / 150 \mathrm{MHz}, \mathrm{DMSO}-d_{6}\right)$ and $2\left(600 / 150 \mathrm{MHz}, \mathrm{CDCl}_{3}\right)$

\begin{tabular}{|c|c|c|c|c|}
\hline \multirow{2}{*}{ No. } & \multicolumn{2}{|l|}{1} & \multicolumn{2}{|c|}{2} \\
\hline & $\delta_{\mathrm{H}}(J$ in $\mathrm{Hz})$ & $\delta_{\mathrm{C}}$ & $\delta_{\mathrm{H}}(J$ in $\mathrm{Hz})$ & $\delta_{\mathrm{C}}$ \\
\hline 2 & & 163.6 & & 164.2 \\
\hline 3 & $6.98 \mathrm{~s}$ & 104.2 & $6.56 \mathrm{~s}$ & 104.2 \\
\hline 4 & & 182.9 & & 182.7 \\
\hline 5 & & 144.6 & & 151.6 \\
\hline $5-\mathrm{OH}$ & $12.35 \mathrm{~s}$ & & $12.74 \mathrm{~s}$ & \\
\hline 6 & & 136.2 & & 136.5 \\
\hline 7 & & 148.1 & & 151.7 \\
\hline 8 & & 130.7 & & 123.1 \\
\hline $8-\mathrm{OH}$ & $9.33 \mathrm{~s}$ & & & \\
\hline 9 & & 141.4 & & 144.5 \\
\hline 10 & & 106.5 & & 106.7 \\
\hline $1^{\prime \prime}$ & & & & 174.4 \\
\hline $2^{\prime \prime}$ & & & $2.80 \sim 2.78 \mathrm{~m}$ & 41.1 \\
\hline $3^{\prime \prime}$ & & & $1.41 \mathrm{~d}(7.2)$ & 17.0 \\
\hline $4^{\prime \prime}$ & & & $\begin{array}{l}1.95 \sim 1.93 \mathrm{~m} \\
1.72 \sim 1.69 \mathrm{~m}\end{array}$ & 26.8 \\
\hline $5^{\prime \prime}$ & & & $1.09 \mathrm{t}(7.6)$ & 11.7 \\
\hline $6-\mathrm{OCH}_{3}$ & $3.83 \mathrm{~s}$ & 60.5 & $3.96 \mathrm{~s}$ & 61.0 \\
\hline 7- $-\mathrm{OCH}_{3}$ & $3.95 \mathrm{~s}$ & 61.2 & $4.02 \mathrm{~s}$ & 61.4 \\
\hline $1^{\prime}$ & & 125.8 & & 123.4 \\
\hline $2^{\prime}$ & $7.31 \mathrm{~d}(1.6)$ & 108.1 & $7.76 \mathrm{~d}(8.8)$ & 128.1 \\
\hline $3^{\prime}$ & & 150.9 & $6.98 \mathrm{~d}(8.8)$ & 114.4 \\
\hline 3'-OH & $9.69 \mathrm{~s}$ & & & \\
\hline $4^{\prime}$ & & 139.8 & & 162.7 \\
\hline $5^{\prime}$ & & 153.5 & $6.98 \mathrm{~d}(8.8)$ & 114.4 \\
\hline $6^{\prime}$ & $7.24 \mathrm{~d}(1.6)$ & 102.3 & $7.76 \mathrm{~d}(8.8)$ & 128.1 \\
\hline $4^{\prime}-\mathrm{OCH}_{3}$ & $3.76 \mathrm{~s}$ & 60.1 & $3.88 \mathrm{~s}$ & 55.5 \\
\hline $5^{\prime}-\mathrm{OCH}_{3}$ & $3.89 \mathrm{~s}$ & 56.0 & & \\
\hline
\end{tabular}

Shimadzu double-beam210A 型紫外分光光度计(日本岛 津) 测定, 熔点由 WRS-2 型熔点仪 (上海仪电物理光学仪 器公司) 测定, 比旋光度由 SEPA-300 数字型旋光仪(日 本 Horiba)测定, 核磁共振数据由 Bruker Avance III-600 测定(德国 Bruker 公司生产), 以 TMS 为内标; 分析型和 制备型 HPLC 分别为 Agilent 1100 HPLC 型和 Agilent 1200 HPLC 型, 检测器为 DAD 检测器, 分析型百为四 元原, 制备型永为二元原, 进样器为自动进样器 (美国 安捷伦公司生产), 分析型色谱柱为 Agilent Zorbax SB-C18; 制备型色谱柱同为 Agilent Zorbax SB-C18; 正 相柱层析硅胶 $\left(80 \sim 100\right.$ 目和 $200 \sim 300$ 目) 以及 $\mathrm{GF}_{254}$ 预 制硅胶板均由青岛海洋化工厂生产; Rp-18 反相硅胶 (40 75 $\mu \mathrm{m})$ 由日本 Fuji silysia 化学公司生产; Sephadex LH-20 为瑞典 Amersham Biosciences 公司产品.

\section{2 植物材料}

全叶马兰药材 2014 年 9 月采于安徽合肥市磨店乡, 经安徽中医药大学中药标本中心杨青山老师鉴定为菊
科马兰属全叶马兰(Kalimeris integrifolia Turcz.ex DC.) 干燥全草. 凭证标本(No. 201409)存放在安徽中医药大 学天然药物化学研究组.

\section{3 提取分离}

全叶马兰干燥地上部分 $13 \mathrm{~kg}, 80 \%$ 乙醇回流提取 3 次, 每次 $2 \mathrm{~h}$, 合并提取液减压浓缩后, 得到 $2568 \mathrm{~g}$ 浸 膏, 直接采用正相硅胶柱层析以二氯甲烷-甲醇为流动 相进行梯度洗脱, 洗脱后共得到 $\mathrm{M} 1 \sim \mathrm{M} 7$ 七个部分. $\mathrm{CH}_{2} \mathrm{Cl}_{2} / \mathrm{MeOH}(V: V=30: 1,20 ： 1)$ 洗脱部位 $\mathrm{M} 4$ 通过 正相硅胶柱层析, 以 $[V$ (石油醚) $: V($ 乙酸乙酯 $=50: 1$, $30: 1,20: 1,15: 1,10: 1,5: 1,3: 1,2: 1,1: 1$ ]梯 度洗脱，薄层色谱(TLC)检识后合并相似流份共得到 4 个组分 $\left(\mathrm{M}_{4} 1 \sim \mathrm{M}_{4} 4\right), \mathrm{M}_{4} 1$ 放置后母液经正相硅胶柱层析 以 $[V$ (石油醚) $: V($ 乙酸乙酯 $=30: 1,20: 1,15: 1,10$ : $1,5: 1,3: 1,2: 1,1: 1]$ 梯度洗脱, TLC 检识后合并相 似流份共得到 7 个组分 $\left(\mathrm{M}_{41} 1 \sim \mathrm{M}_{41} 7\right), \mathrm{M}_{41} 4$ 经正相硅胶 柱层析 $[V$ (石油醚) $: V$ (丙酮 $)=15: 1,10: 1,5: 1,3: 1$, $2: 1,1: 1]$ 梯度洗脱, TLC 检识后合并相似流份共得到 9 个组分 $\left(\mathrm{M}_{414} 1 \sim \mathrm{M}_{414} 9\right), \mathrm{M}_{414} 3$ 放置后析出以黄色沉淀, 将沉淀与母液分离后, 沉淀溶解后, 葡聚糖凝胶柱层析 纯化后得到化合物 2 (316.7 mg); $\mathrm{M}_{43}$ 经正相硅胶柱层 析 $[V($ 石油醚 $): V($ 丙酮 $)=15: 1,10: 1,5: 1,3: 1,2$ : 1, 1：1]梯度洗脱, TLC 检识后合并相似流份共得到 9 个 组分 $\left(\mathrm{M}_{435} 1 \sim \mathrm{M}_{435} 9\right), \mathrm{M}_{435} 6$ 葡聚糖凝胶柱层析纯化后得 到化合物 1 (30.6 mg).

Kalinturflavone A (1): 黄色针状晶体, 熔点 251 $253{ }^{\circ} \mathrm{C} ;{ }^{1} \mathrm{H}$ NMR 和 ${ }^{13} \mathrm{C}$ NMR 数据见表 1; UV $(\mathrm{MeOH})$ $\lambda_{\max }\left[\lg \varepsilon /\left(\mathrm{L} \cdot \mathrm{mol}^{-1} \cdot \mathrm{cm}^{-1}\right)\right]: 306$ (4.35), 207 (4.52) nm; IR (KBr) $v_{\max }: 3419,1657,1615,1594,1485,1458,1430$, 1387, 1231, 1106, 1031, 1000, 960, $845 \mathrm{~cm}^{-1}$; HR-EI-MS calcd for $\mathrm{C}_{19} \mathrm{H}_{17} \mathrm{O}_{9}\left([\mathrm{M}-\mathrm{H}]^{-}\right.$389.0878, found 389.0894.

Kalinturflavone B (2): 黄色粉末, 熔点 110 $112{ }^{\circ} \mathrm{C},[\alpha]_{\mathrm{D}}^{19}-3.4\left(c \quad 0.013, \mathrm{CHCl}_{3}\right) ;{ }^{1} \mathrm{H}$ NMR 和 ${ }^{13} \mathrm{C}$ NMR 数据见表 $2 ; \mathrm{UV}\left(\mathrm{CHCl}_{3}\right) \lambda_{\max }\left[\mathrm{lg} \varepsilon /\left(\mathrm{L} \cdot \mathrm{mol}^{-1}\right.\right.$ • $\left.\mathrm{cm}^{-1}\right)$ ]: 327 (4.30), 283 (4.23) nm; IR (KBr) $v_{\max }$ : 3443, $3427,2969,2937,1756,1657,1603,1577,1508,1487$, 1463, 1431, 1376, 1262, 1220, 1184, 1114, 1067, 1030, $1012,834 \mathrm{~cm}^{-1}$; HR-ESI-MS calcd for $\mathrm{C}_{23} \mathrm{H}_{23} \mathrm{O}_{8}[\mathrm{M}-\mathrm{H}]^{-}$ 427.1398, found 427.1399.

辅助材料(Supporting Information) 新化合物 1 和 2 的 UV, IR 谱, HR-EIMS 谱, 1D NMR 和 2D NMR 谱图. 这 些材料可以免费从本刊网站(http://sioc-journal.cn/)上下 载. 


\section{References}

[1] Chinese Flora Editorial Board of Chinese Academy of Sciences Flora of China, Vol. 74, Science Press, Beijing, 1985 (in Chinese). (中国科学院中国植物志编辑委员, 中国植物志, 第 74 卷, 科学 出版社, 北京, 1985.)

[2] Chinese Herbal Editorial Board Chinese Herbal, Shanghai Science and Technology Press, Shanghai, 1999 (in Chinese). (国家中医药管理局《中华本草》编委会, 中华本草, 上海科学 技术出版社，上海, 1999.)

[3] Ji, P.; Wang, G. K.; Liu, J. S.; Luo, Z. H.; Liu, J. K.; Wang, G. Nat. Prod. Res. Dev. 2014, 26, 212 (in Chinese).

(季鹏, 王国凯, 刘劲松, 罗争辉, 刘吉开, 王刚, 天然产物研究 与开发, 2014, 26, 212.)

[4] Zhong, W. W.; Liu, J. S.; Zhang, C. E.; Chen A. M.; Xu, Y. S.; Wang G. Guihaia 2012, 32, 261 (in Chinese).

(钟文武, 刘劲松, 张聪佴, 陈爱民, 许应生, 王刚, 广西植物, 2012, 32, 261.)

[5] Wang, G. K.; Liu, J. S.; Zhang, C. E.; Wang, Z.; Cai, B. X.; Wang, G. Chin. Med. Mater. 2015, 38, 82 (in Chinese). (王国凯, 刘劲松, 张聪佴, 王净, 蔡百祥, 王刚, 中药材, 2015, $38,82$.

[6] Liu, J. S.; Wang G.; Wang, G. K.; Yu, B.; Wang, F.; Liu, J. K. Chin.
Trad. Pat. Med. 2010, 32, 462 (in Chinese)

(刘劲松, 王国凯, 王刚, 余波, 王飞, 刘吉开, 中成药, 2010, 32, 462.)

[7] Zhang, J.; Shen, P.; Lu, T.; Yu, D.; Li, H.; Yang, G. Z. Acta Chim. Sinica 2011, 69, 383 (in Chinese).

(张骥, 申鹏, 陆涛, 余丹妮, 李卉, 杨国忠, 化学学报, 2011，69, 383.)

[8] Tchinda, A. T.; Mouokeu, S. R.; Ngono, R. A.; Ebelle, M. R.; Mokale, A. L.; Nono, D. K.; Frédérich, M. Nat. Prod. Res. 2015, 29, 1990.

[9] Bui, M. L.; Grayer, R. J.; Veitch N. C.; Kiteb, G. C.; Trana, H.; Nguyena, Q. K. Biochem. Syst. Ecol. 2004, 32, 943.

[10] Fushiya, S.; Kishi, Y.; Hattori, K.; Batkhuu J.; Takano, F.; Singab, A. N.; Okuyama, T. Planta Med. 1999, 65, 404.

[11] Parmar, V. S.; Bisht, K. S.; Sharma, S. K.; Jain, R.; Taneja, P.; Singh, S.; Simonsen, O.; Boll, P. M. Phytochemistry 1994, 36, 507.

[12] Xu, N. S.; Yang, H. M.; Cui, M.; Song, F. R.; Liu, Z. Q.; Liu, S. Y. Chin. J. Chem. 2012, 30, 1433.

[13] Zhang, Y. L.; Jiang, L. L.; Xiao, T. S.; Li, Y. B. J. Asian. Nat. Prod. Res. 2015, 17, 717.

[14] Maldonado, E.; Toscano, R. A.; Mancera, C.; Tripp, M. T.; Ortega, A. Phytochemistry 1992, 31, 1003.

(Zhao, X.) 